\title{
Nuclear factor of activated $T$ cells mediates oxidised LDL-induced calcification of vascular smooth muscle cells
}

\author{
C. Goettsch • M. Rauner • C. Hamann • K. Sinningen • \\ U. Hempel • S. R. Bornstein • L. C. Hofbauer
}

Received: 12 April 2011 / Accepted: 23 May 2011 /Published online: 24 June 2011

(C) Springer-Verlag 2011

\begin{abstract}
Aims/hypothesis Vascular calcification is a prominent feature of both atherosclerosis and diabetes, and is clinically associated with osteoporosis. The expression of bone-regulatory factors and the impact of oxidative stress in aortic calcification are well-documented. Recently, nuclear factor of activated T cells (NFAT) cytoplasmic, calcineurin-dependent 1 (NFATc1) was identified in calcified aortic valves and has been implicated in vascular calcification. Therefore, we assessed the mechanisms of osteogenic transdifferentiation of vascular smooth muscle cells induced by oxidised LDL (oxLDL) and evaluated the role of NFAT in this process.
\end{abstract}

Electronic supplementary material The online version of this article (doi:10.1007/s00125-011-2219-0) contains peer-reviewed but unedited supplementary material, which is available to authorised users.

C. Goettsch • M. Rauner $\cdot$ K. Sinningen $\cdot$ S. R. Bornstein •

L. C. Hofbauer

Division of Endocrinology, Diabetes and Metabolic

Bone Diseases, Department of Medicine III,

Technical University Medical Center,

Fetscherstraße 74,

01307 Dresden, Germany

C. Hamann

Department of Orthopaedics, Technical University,

Dresden, Germany

\section{U. Hempel}

Institute of Physiological Chemistry,

Technical University,

Dresden, Germany

S. R. Bornstein • L. C. Hofbauer $(\square)$

Center for Regenerative Therapies Dresden, Technical University,

Dresden, Germany

e-mail: lorenz.hofbauer@uniklinikum-dresden.de
Methods Human coronary artery smooth muscle cells (HCASMCs) were cultured for 21 days in medium supplemented with oxLDL. NFAT was inhibited using the NFAT inhibitor VIVIT, or by knockdown with small interfering RNA (siRNA). Osteogenic transdifferentiation was assessed by gene expression, matrix mineralisation and alkaline phosphatase activity.

Results Exposure to oxLDL caused the transformation of HCASMCs towards an osteoblast-like phenotype based on increased mineral matrix formation and RUNX2 expression. NFATc1 blockade completely prevented oxLDL-induced osteogenic transformation of HCASMCs as well as oxLDL-induced stimulation of osteoblast differentiation. In contrast, matrix mineralisation induced by osteogenic medium was independent of the NFAT pathway. Of note, oxLDL-conditioned medium from HCASMCs transferred to bone cells promoted osteoblast mineralisation. Consistent with these in vitro findings, diabetic rats with a twofold increase in oxidised lipid levels displayed higher aortic calcium concentrations and increased expression of osteogenic markers and production of NFATc1.

Conclusions/interpretation Our results identify the NFAT signalling pathway as a novel regulator of oxLDL-induced transdifferentiation of vascular smooth muscle cells towards an osteoblast-like phenotype.

Keywords Diabetes - Matrix mineralisation - NFAT. Osteogenic transformation - Oxidative stress - oxLDL . RUNX2 · Vascular calcification

$\begin{array}{ll}\text { Abbreviations } \\ \text { BMSC } & \text { Bone marrow stromal cell } \\ \text { HCASMC } & \text { Human coronary artery smooth muscle cell } \\ \text { NAC } & N \text {-acetyl cysteine } \\ \text { NFAT } & \text { Nuclear factor of activated T cells }\end{array}$




$\begin{array}{ll}\text { nLDL } & \text { Native LDL } \\ \text { OM } & \text { Osteogenic medium } \\ \text { oxLDL } & \text { Oxidised LDL } \\ \text { RANKL } & \text { Receptor activator of NFkB ligand } \\ \text { ROS } & \text { Reactive oxygen species } \\ \text { VSMC } & \text { Vascular smooth muscle cell } \\ \text { WT } & \text { Wild type } \\ \text { ZDF rat } & \text { Zucker diabetic fat rat }\end{array}$

\section{Introduction}

Vascular calcification is a prominent feature of chronic diseases such as diabetes mellitus and atherosclerosis. Each of the individual components of the metabolic syndrome, hyperlipoproteinaemia, diabetes mellitus and arterial hypertension, are independent risk factors for vascular calcification. Crosstalk between the immune system, the vascular system and bone metabolism plays an important role in the pathogenesis of vascular calcification [1,2]. Medial artery calcification, as in Mönckeberg's sclerosis and atherosclerotic calcification, which is found in the intima of the vascular wall, is prevalent in patients with type 2 diabetes [3]. Clinically, there is a correlation between osteoporosis and vascular calcification. Various in vitro and in vivo studies suggest that cellular and molecular processes in vascular calcification are similar to those seen in pathological bone remodelling. Recently, we demonstrated a negative correlation between bone loss and vascular calcium content and a positive correlation between markers of bone resorption and vascular calcium content in mice $[4,5]$. A nuclear magnetic resonance-based study reported striking similarities between mineral formed by bone cells and mineralised atherosclerotic plaques [6]. Moreover, osteoblast- and osteoclast-like cells and various bone-related proteins have been detected in areas of vascular calcification and within atherosclerotic lesions [7, 8]. Recently, enhanced osteoblastic activity has been reported to occur in aortas undergoing early stages of atherosclerosis [9]. Moreover, osteoblast-derived factors such as receptor activator of nuclear factor $\mathrm{KB}(\mathrm{NF} \kappa \mathrm{B})$ ligand (RANKL) and osteoprotegerin represent potent mediators of the vascular system [10]. Various studies provide evidence of transdifferentiation of vascular smooth muscle cells (VSMCs), which is a key feature in vascular calcification and is promoted in vitro by using $\beta$-glycerol phosphate [11], TNF $\alpha$ [12], or interleukin-4 [13] and in vivo using matrix Gla protein-deficient mice [14].

Oxidative stress is an established risk factor for vascular disease. Specific NADPH oxidase complexes have been described as a major source for generating reactive oxygen species (ROS) in various types of vascular cell $[15,16]$. An impaired balance between ROS formation and antioxidative mechanisms results in cellular oxidative stress. In vascular cells, increased oxidative stress reduces the availability of nitric oxide, potentiates redox-sensitive signal transduction, and is able to convert LDL into oxidised (ox)LDL, the latter representing a pivotal established risk factor for cardiovascular disease. Interestingly, enhanced circulating levels of oxLDL have been found in patients with impaired glucose tolerance [17] and type 2 diabetes [18]. Furthermore, oxidative stress and oxidised lipids may promote osteogenic differentiation under appropriate conditions [19-22].

The nuclear factor of activated $\mathrm{T}$ cells (NFAT) is a transcription factor originally described in immune cells. NFAT is located in the cytoplasm and translocates into the nucleus upon activation [23]. Oxidative stress mediated by oxLDL activates NFAT in T lymphocytes [24]. Recently, vascular smooth muscle cells were found to mainly express NFAT cytoplasmic, calcineurin-dependent 1 (NFATc1) and NFATc2 and, to a smaller extent, NFATc3 [25]. Moreover, NFATc1 was identified in calcified aortic valves, indicating its involvement in the calcification process [26]. However, the role of the NFAT pathway in osteogenic transdifferentiation during vascular calcification remains poorly defined. Here, we aimed to study the role of the NFAT pathway in osteogenic transdifferentiation of smooth muscle cells initiated by oxLDL.

\section{Materials and methods}

Cell culture All cell culture reagents were purchased from Invitrogen (Karlsruhe, Germany). The chemicals were from Sigma-Aldrich (Taufkirchen, Germany) unless otherwise specified. Human coronary artery smooth muscle cells (HCASMCs) were purchased from Promocell (Heidelberg, Germany). HCASMCs were grown in Smooth Muscle Cell Growth Medium 2 (SMC-GM2) from Promocell supplemented with epidermal growth factor $(0.5 \mathrm{ng} / \mathrm{ml})$, insulin $(5 \mu \mathrm{g} / \mathrm{ml})$, basic fibroblast growth factor-B $(2 \mathrm{ng} / \mathrm{ml})$ and fetal bovine serum (5\%). The cells were maintained at $37^{\circ} \mathrm{C}\left(5 \% \mathrm{CO}_{2}, 90 \%\right.$ humidity) and were used between passages 4 and 8 . Cells isolated from three to five independent donors were used.

Transfection To inhibit the expression of NFATc1, ONTARGETplus SMART-pool (L-003605-00-0010) and a negative control from Dharmacon RNAi Technologies (Chicago, IL, USA) were used. Transfection of $50 \mathrm{nmol} / 1 \mathrm{siRNAs}$ was performed using DharmaFECT 1 transfection reagent twice per week over the entire cell culture period [27].

LDL isolation Native LDL (nLDL) and oxLDL were isolated and characterised by standard procedures as described [16]. In brief, VLDL, LDL and HDL were separated by centrifugation through $\mathrm{NaBr}$ gradients from human plasma. The LDL fraction was dialysed against PBS 
and oxidised using $50 \mu \mathrm{mol} / 1 \mathrm{CuSO}_{4}$. The oxidation level of every LDL was analysed by gel electrophoresis (Hydragel LDL/HDL CHOL Direct K20 kit, Sebia, Norcross, GA, USA) and measurement of conjugated dienes (see electronic supplementary material [ESM] Table 1). Confluent cultures of HCASMCs were stimulated with oxLDL or nLDL as control for the indicated times (up to 21 days) and concentrations (up to $100 \mu \mathrm{g} / \mathrm{ml}$ ). Up to four different LDL isolations and independent oxidations were used.

NFATc1 activation assay Nuclear protein extracts were isolated from HCASMC cultures using the Nuclear Extract Kit (Active Motif, Rixensart, Belgium). The activation of NFATc1 was measured using the TransAM NFATc1 kit (Active Motif) according to the manufacturer's protocol. In brief, $8 \mu \mathrm{g}$ nuclear protein was incubated for $1 \mathrm{~h}$ in a 96well plate coated with an oligonucleotide containing an NFAT consensus core sequence (5'-T/AGGAAA-3'). After NFATc1 binding and subsequent washing steps, NFATc1 antibody (1:500 dilution) was added.

RNA preparation and real-time PCR Total RNA from the cell culture was isolated using the High Pure RNA Isolation Kit (Roche, Mannheim, Germany) and from the rat aorta using RNeasy Fibrous Tissue Mini Kit from Qiagen (Hilden, Germany), both according to the manufacturer's protocols. The mRNA expression was determined by SYBR greenbased real-time PCR reactions using a standard protocol (Roche). The primer pairs used are summarised in ESM Table 2. The expression was normalised to $\beta$-actin. The results were calculated using the $\Delta \Delta C_{t}$ method, and are presented in $x$-fold increase relative to control.

Immunofluorescence After treatment with oxLDL, cells were washed with PBS and fixed in 4\% paraformaldehyde for $15 \mathrm{~min}$. Cells were permeabilised for $10 \mathrm{~min}$ in $0.5 \%$ Triton X-100. Subsequently, cells were washed and blocked with $1 \%$ BSA in PBS for $30 \mathrm{~min}$. The glass slides were exposed to an anti-NFATc1 antibody (Santa Cruz, Heidelberg, Germany) overnight (1:100) and incubated for $1 \mathrm{~h}$ with an Alexa Fluor 488-labelled secondary antibody (Invitrogen). After three washing steps, cells were stained with DAPI for $5 \mathrm{~min}$ and washed again for $15 \mathrm{~min}$. Thereafter, glass slides were embedded in mounting medium (Dako, Glostrup, Denmark) and examined using a Zeiss Axio Imager M.1 fluorescence microscope (Zeiss, Jena, Germany).

Osteogenic transdifferentiation of HCASMCs HCASMCs were cultured for up to 21 days in the presence of either regular SMC-GM2 medium with reduced serum $(0.1 \%)$ or osteogenic medium $(\mathrm{OM})$, which consisted of regular medium with $0.1 \%$ serum supplemented with $10 \mathrm{nmol} / 1$ dexamethasone, $10 \mathrm{mmol} / \mathrm{l} \beta$-glycerol phosphate, and $100 \mu \mathrm{mol} / \mathrm{l} \mathrm{L}$ - ascorbate phosphate. Medium was changed three times per week, and $\mathrm{nLDL}$ or oxLDL $(50 \mu \mathrm{g} / \mathrm{ml})$ was replaced. Conditioned medium was collected and stored at $-80^{\circ} \mathrm{C}$. The NFAT inhibitor VIVIT $(1 \mu \mathrm{mol} / 1$, Calbiochem, Germany) and the radical scavenger $N$-acetyl cysteine (NAC, $500 \mu \mathrm{mol} / 1$, Sigma-Aldrich) were also added at every medium change.

Mineralisation assay and activity of alkaline phosphatase Mineralised matrix formation was assessed by Alizarin Red $\mathrm{S}$ staining at different time points. HCASMCs were fixed in 4\% PFA and stained with $40 \mathrm{mM}$ Alizarin Red S (pH 4.2) for $30 \mathrm{~min}$ at room temperature. Excess dye was removed by washing the plates with distilled water. Incorporated calcium was eluted with $100 \mathrm{mmol} / 1$ cetylpyridinium chloride and measured at $540 \mathrm{~nm}$. Alkaline phosphatase (ALP) activity was measured in cell cultures as previously described [27].

Cell viability Cell viability was assessed using a commercially available kit (Cell Titer Blue, Promega, Mannheim, Germany) which measures the amount of fluorescent resorufin that has been reduced in the mitochondria of viable cells from resazurin. Briefly, after a cultivation period of 21 days with $10-100 \mu \mathrm{g} / \mathrm{ml}$ oxLDL or OM, $20 \mu \mathrm{l}$ substrate per $100 \mu \mathrm{l}$ medium was added and the cells were incubated for $2 \mathrm{~h}$ at $37^{\circ} \mathrm{C}$. Finally, the fluorescence intensity was quantified in duplicate using the FluoStar Omega (Ex, $560 \mathrm{~nm}$; Em, $590 \mathrm{~nm}$; BMG LABTECH, Offenburg, Germany).

Osteoblast mineralisation Primary human bone marrow stromal cells (BMSCs) were kindly provided by the Department of Medicine I of the Dresden University Medical Center and cultured according to previously reported methods [28]. BMSCs were maintained in DMEM, 10\% fetal calf serum (Supreme, Lonza, Cologne, Germany) and $1 \%$ penicillin/streptomycin in a humidified atmosphere of $95 \%$ air and $5 \% \mathrm{CO}_{2}$. Cells in passages 5 were used. To induce osteogenic differentiation, cells were cultured in growth medium supplemented with L-ascorbate phosphate $(100 \mu \mathrm{mol} / \mathrm{l}), \beta$-glycerol phosphate $(10 \mathrm{mmol} / \mathrm{l})$ and dexamethasone $(10 \mathrm{nmol} / \mathrm{l})$. Mature osteoblasts were obtained after 21 days of culture. To assess the interaction of HCASMCs and osteoblastogenesis, BMSCs were cultured in medium containing equivalent quantities of osteoblast differentiation medium and the HCASMC culture supernatant fraction, obtained after $\mathrm{nLDL}$ or oxLDL exposure, containing the final concentrations of differentiation factors, as detailed above. Conditioned medium obtained from untreated cells and fresh medium, which had never been used for cell culture, served as controls. Mineralised matrix was assessed as described above. 
Zucker diabetic fat rat model All animal procedures were approved by the Institutional Review Board of the University of Dresden (24D-9168.11-1/2008-30) and followed the principles of laboratory animal care. Wild type (WT; ZDF/GmiCrl-Lepr ${ }^{+/+}$) and Zucker diabetic fat (ZDF; $\mathrm{ZDF} / \mathrm{GmiCrl}-$ Lepr $^{\mathrm{fa} / \mathrm{fa}}$ ) rats were purchased from Charles River Laboratories (Wilmington, MA, USA). Male rats (22 weeks old; $n \geq 9$ each) were used to isolate the thoracic aorta. Blood samples were taken and lipid variables and glucose levels were measured. VSMCs were isolated using the explant technique. Briefly, the thoracic aorta was isolated under sterile conditions and cut longitudinally. The endothelium was removed by scraping off the cell layer with a sterile scalpel. The aorta was cut into $2-\mathrm{mm}$ pieces and its luminal surface was placed in contact with the culture flask wall of a well plate. After 1 week, VSMCs had migrated and aorta specimens were removed. VSMCs were used between passages 2 and 4 . The VSMC phenotype was confirmed by the expression of $\alpha$-smooth muscle actin using immunofluorescence. Osteogenic transdifferentiation was induced as described above. The calcium (Calcium Liquicolor kit) and phosphate $\left(\mathrm{NH}_{4}\right.$ molybdate-based assay) content of the aorta were measured using standard assays from Greiner Diagnostics (Bahlingen, Germany) as previously described [5]. Mineral content in nanomoles was normalised to the weight of the aortic specimen in milligrams.

Statistical analysis Data are given as means \pm SEM, and $n$ indicates the number of independent experiments. Statistical analysis for time-response curves were performed using a one-way ANOVA with Bonferroni's post hoc test, differences between the groups from time kinetics using a twoway ANOVA with Bonferroni's post hoc test, and single group comparisons using a Student's $t$ test. Correlation analyses were performed according to Pearson (SPSS 17.0, Chicago, IL, USA). A value of $p<0.05$ was considered statistically significant.

\section{Results}

oxLDL-induced osteogenic transdifferentiation of HCASMCs Exposure of HCASMCs to oxLDL (10-
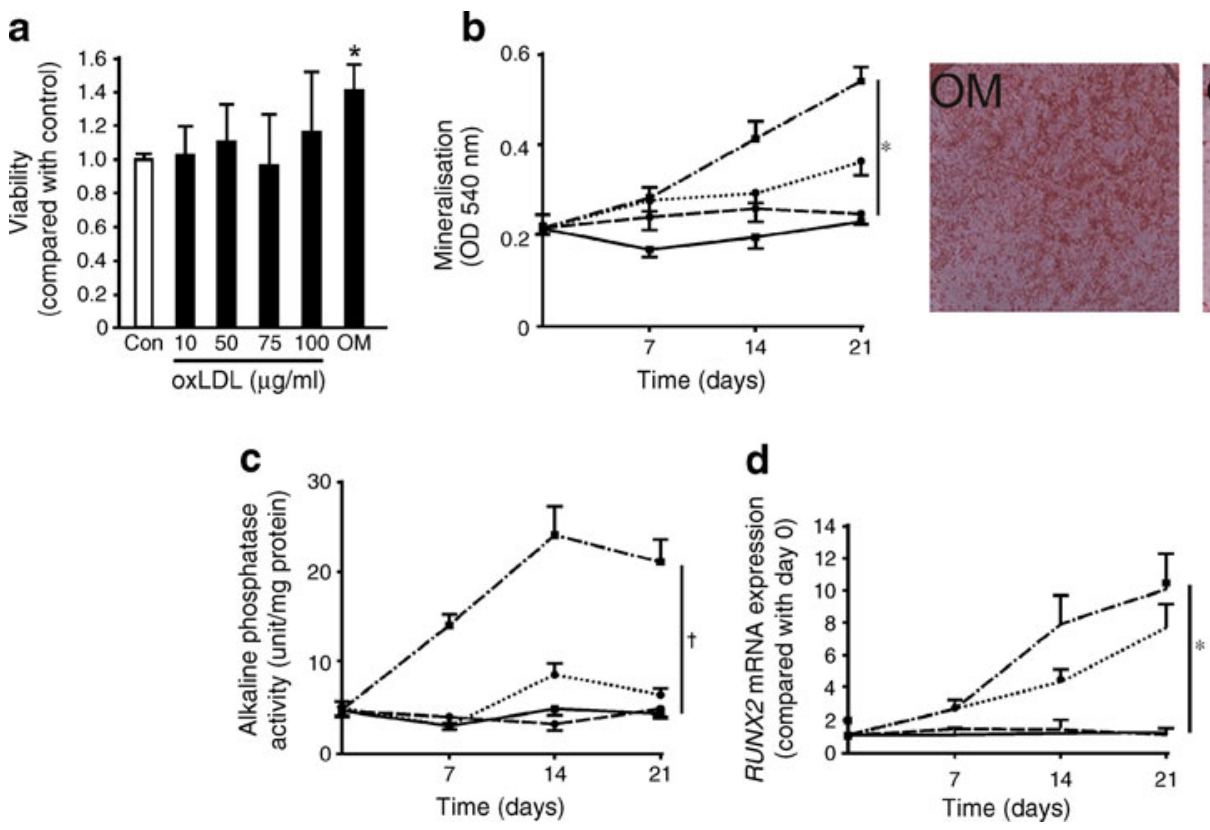

e

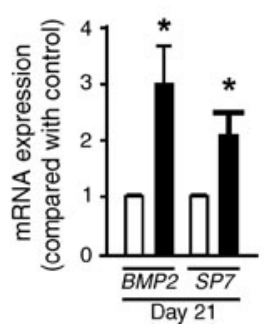

Fig. 1 oxLDL promotes osteogenic transdifferentiation of HCASMCs. a Cell viability: HCASMCs were cultured for 21 days in control medium (con), control medium supplemented with oxLDL $(10-100 \mu \mathrm{g} / \mathrm{ml})$, or OM; $n=3,{ }^{*} p<0.05$ vs con. b-e HCASMCs were cultured for 7, 14 and 21 days in control medium, control medium supplemented with $50 \mu \mathrm{g} / \mathrm{ml} \mathrm{nLDL}$ or oxLDL, or $\mathrm{OM}$ as positive control; ${ }^{*} p<0.05$; for $\mathbf{b}-\mathbf{d}$, solid line, con; dashed/dotted line $\mathrm{OM}$; dotted line, oxLDL; dashed line, nLDL. b Matrix mineralisation: $n=$ 4-7; one representative picture is shown. The quantification is summarised in the corresponding line graphs (one-way ANOVA: con, $p<0.05$; nLDL, $p=0.702$; oxLDL, $p<0.01$; OM, $p<0.01$. c ALP activity: $n=4-6$. The quantification is summarised in the corresponding line graphs (one-way ANOVA: con, $p<0.232$; nLDL, $p=0.431$; oxLDL, $p<0.01$; OM, $p<0.01$. d RUNX2 mRNA expression: $n=3-7$. The quantification is summarised in the corresponding line graphs (one-way ANOVA: con, $p<0.105$; nLDL, $p=0.186$; oxLDL, $p<0.01$; OM, $p<0.01 .{ }^{*} p<0.05$ con vs oxLDL and OM by two-way ANOVA. e mRNA expression of $B M P 2$ and $S P 7$ in HCASMCs cultured for 21 days with oxLDL; $n=4-6, * p<0.05$; white bars, con; black bars, oxLDL. Results are given as mean \pm SEM 
$100 \mu \mathrm{g} / \mathrm{ml}$ ) over 21 days did not alter cell viability as compared with control cells (Fig. 1a). Treatment of HCASMCs with oxLDL enhanced mineralised matrix formation in a time-dependent manner $(p=0.002$ by ANOVA), and was significantly higher at each time point compared with the untreated control. HCASMCs cultured in normal medium or supplemented with nLDL over 21 days did not form a mineralised matrix (Fig. 1b). As expected, HCASMCs cultured in OM resulted in a marked time-dependent increase in mineralisation compared with cells cultured in control medium. ALP activity, which is an earlier marker of osteogenic differentiation, was significantly increased in cells exposed to oxLDL, while nLDL had no effect (Fig. 1c). The maximum effect was reached at day 14 with a 1.9 -fold increase compared with day $0(p=0.01)$ and a 2.7-fold increase compared with the time-matched
nLDL control $(p=0.001)$. OM significantly increased ALP activity in a time-dependent manner with a maximum effect at day 14 .

To confirm the osteogenic transdifferentiation triggered by oxLDL at the transcriptional level, we set out to assess the expression of the master osteogenic transcription factor gene, $R U N X 2$. After 7 days of exposure, oxLDL significantly induced RUNX2 mRNA levels. After 21 days, $R U N X 2$ mRNA levels were significantly increased by up to eightfold compared with the time-matched control and nLDL control (Fig. 1d). The mRNA expression of RUNX2 was not altered when HCASMCs were cultured in normal medium or in medium supplemented with nLDL over 21 days (Fig. 1d). As expected, OM caused a marked time-dependent upregulation of RUNX2 mRNA levels. Furthermore, we analysed the expression of additional osteoblastic markers;
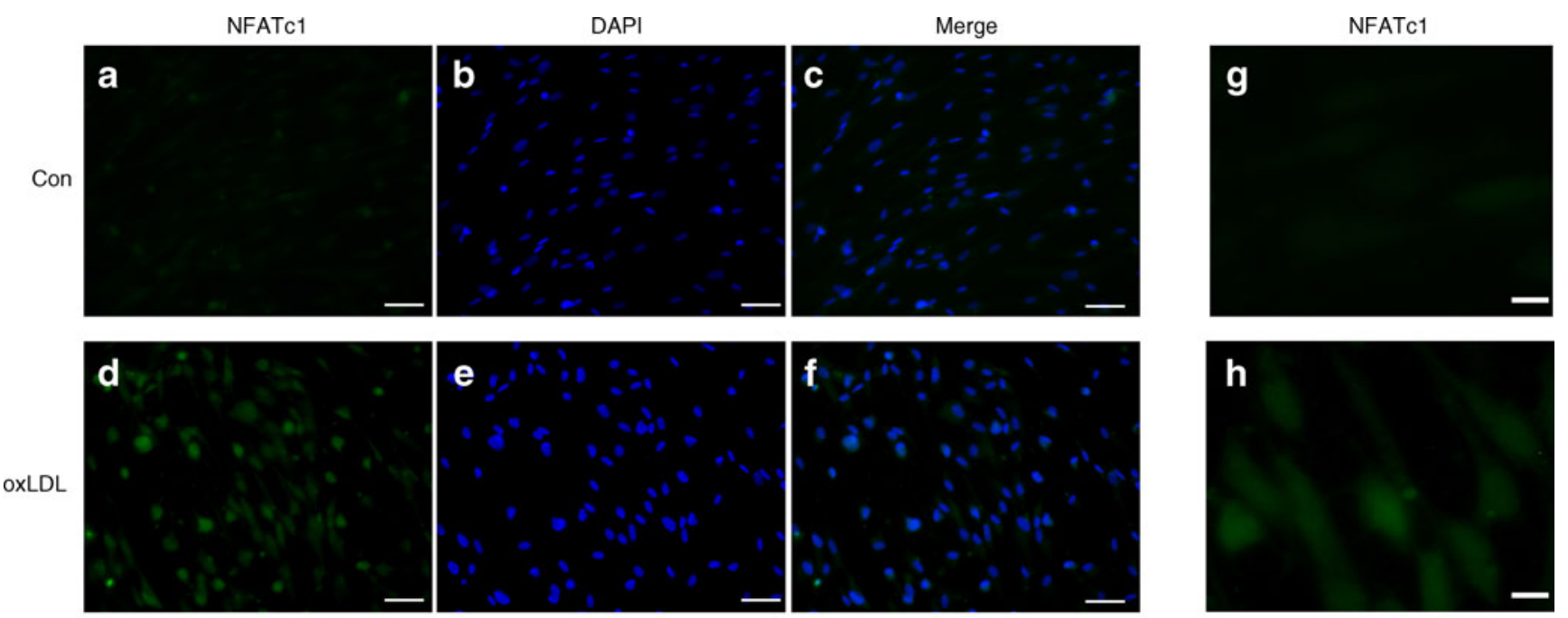

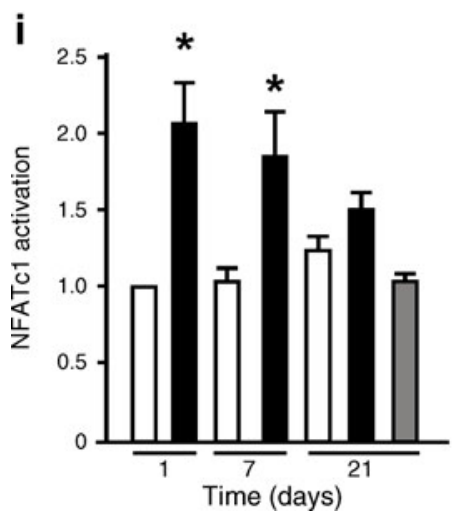

j

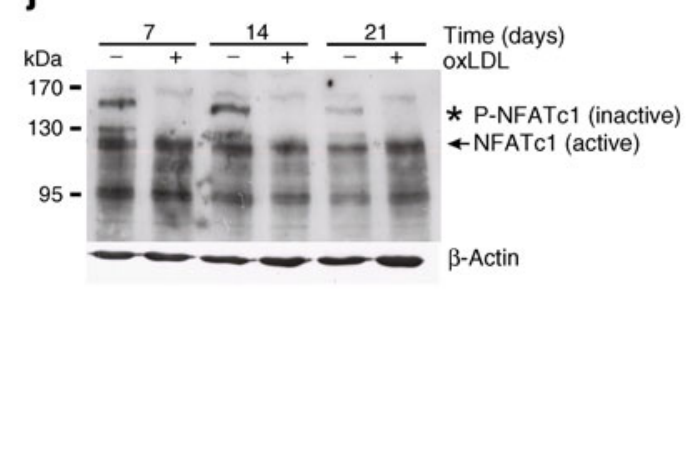

$\mathbf{k}$

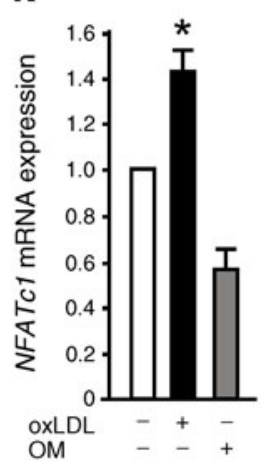

I

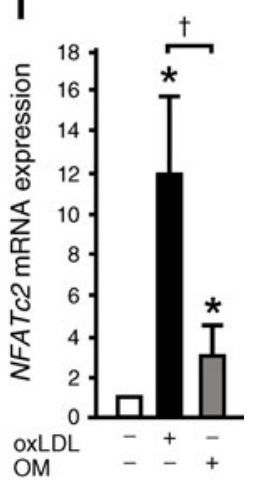

Fig. 2 NFATc1 is regulated during oxLDL-induced HCASMC calcification. a-h HCASMCs were stimulated with $100 \mu \mathrm{g} / \mathrm{ml}$ oxLDL for $24 \mathrm{~h}$. Localisation of NFATc1 (green) was determined by immunofluorescence. Representative images of three independent experiments are shown. a-f: bar, $50 \mu \mathrm{m} ; \mathbf{g}, \mathbf{h}:$ bar, $10 \mu \mathrm{m}$. i HCASMCs were cultured for 1,7 and 21 days in control medium (con), control medium supplemented with $50 \mu \mathrm{g} / \mathrm{ml}$ oxLDL, or OM. NFATc1 activation was assessed using nuclear extracts and NFATc1 TransAM ELISA. $n=3-5 ;{ }^{*} p<0.05$ vs time-matched control; white bars, con; black bars, oxLDL; grey bars, OM. j HCASMCs were cultured for 7, 14 and 21 days in control medium (con), or control medium supplemented with $50 \mu \mathrm{g} / \mathrm{ml}$ oxLDL. NFATc1 protein production: arrow, active, dephosphorylated NFATc1; *inactive, phosphorylated NFATc1; one of three Western blots is shown. k,l HCASMCs were cultured for 21 days in control medium, control medium supplemented with $50 \mu \mathrm{g} / \mathrm{ml}$ oxLDL, or OM. NFATC1 (k) and NFATc2 (l) mRNA expression were analysed using real-time PCR: $n=3-6 ;{ }^{*} p<0.05$ vs time-matched control; ${ }^{\dagger} p<0.05$ 
exposure of oxLDL significantly increased $B M P 2$ and $S P 7$ mRNA expression in calcified HCASMCs (Fig. 1e).

Involvement of the NFAT pathway in oxLDL-induced osteogenic transdifferentiation of HCASMC and similarities with osteoblast mineralisation Because the transcription factor NFAT, a known mediator and activated target of ROS, is a putative factor involved in calcifying HCASMCs, we first analysed the activation of NFAT by oxLDL. oxLDL caused nuclear translocation of NFATc1 in HCASMCs (Fig. 2a-h). In addition, nuclear NFATc1 levels were increased 2.0-fold after 1 and 7 days measured by TransAM ELISA (Fig. 2i). The activation of NFATc1 was also confirmed by Western blot (Fig. 2j). Long-term stimulation with oxLDL caused a decrease in the inactive, phosphorylated form of NFATc1, while the dephosphorylated, active form was increased, which was shown by slower migration within the gel. There was no alteration in the mRNA expression of NFATC1 after oxLDL-stimulation for $24 \mathrm{~h}$ (C. Goettsch, unpublished observation), whereas long-term stimulation significantly induced NFATC1 and NFATC2 expression (Fig. 2k, 1). In contrast, NFATC1 and NFATc2 were not altered in calcified HCASMCs grown in OM.

To assess the role of NFAT in vascular calcification, we used the highly specific 16-mer peptide VIVIT as an inhibitor of the calcineurin-NFAT interaction [29]. Inhibition of NFAT activity completely blocked matrix mineralisation (Fig. 3a) and RUNX2 expression (Fig. 3b) induced by oxLDL. Interestingly, while the induction of RUNX2 expression by OM was inhibited by VIVIT (Fig. 3b), OM-stimulated matrix mineralisation was not altered (Fig. 3a). To further confirm these findings, we knocked down NFATcl using a
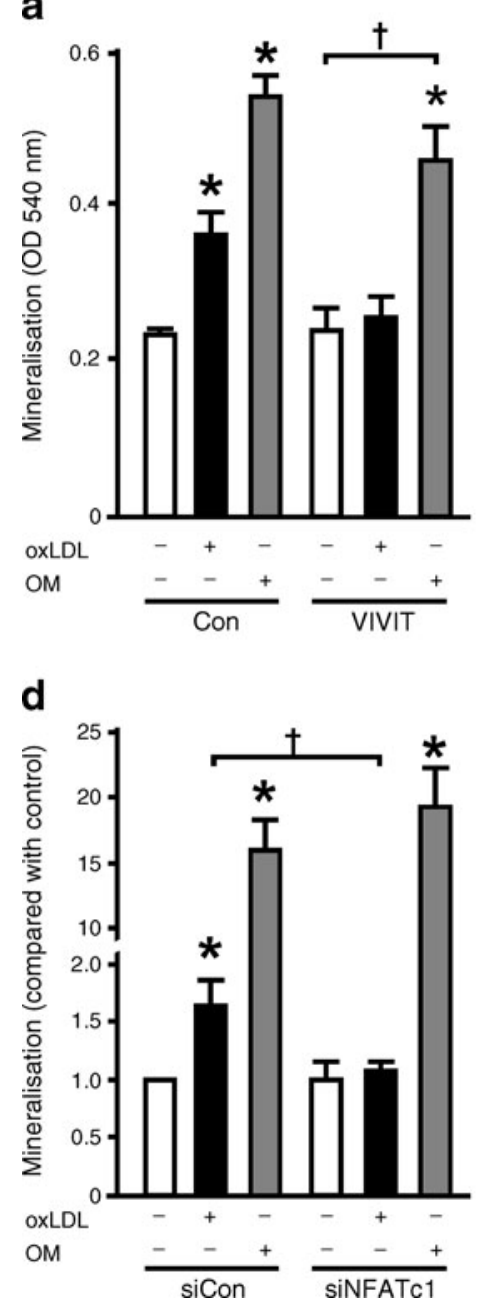

b

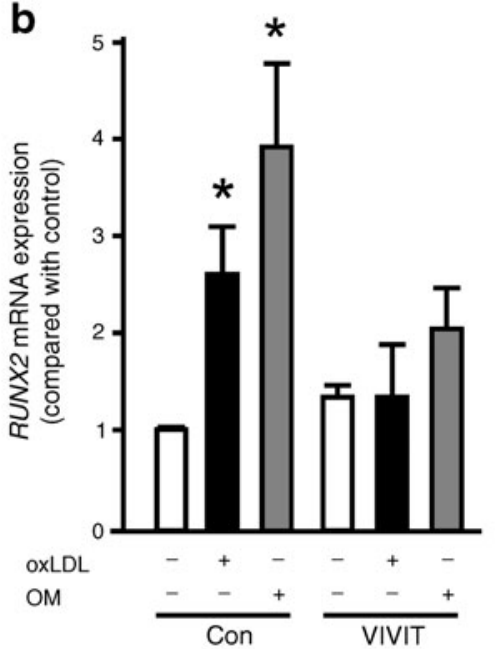

e

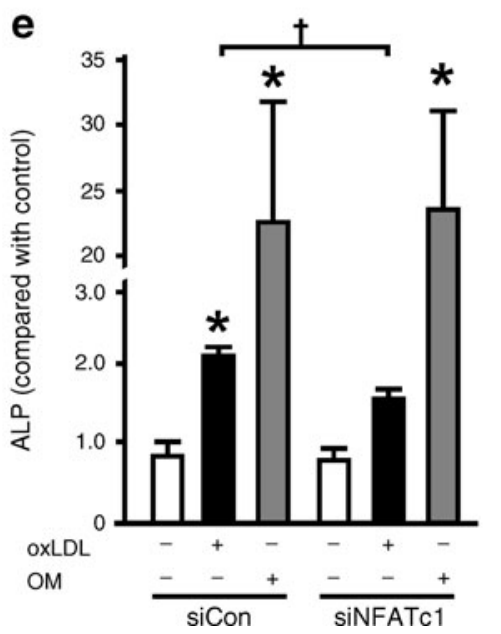

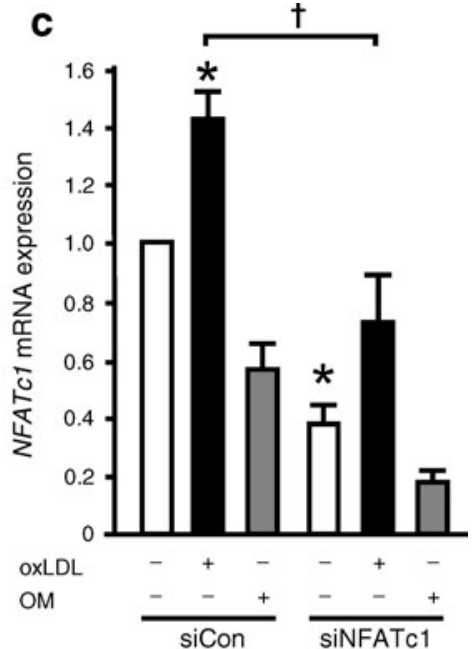

f

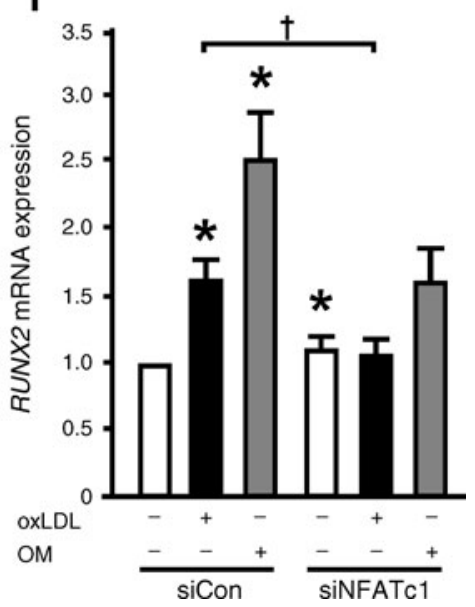

Fig. 3 The NFAT pathway is involved in oxLDL-induced matrix mineralisation and RUNX2 expression in HCASMCs. HCASMCs were cultured for 21 days in control medium (con), control medium supplemented with $50 \mu \mathrm{g} / \mathrm{ml}$ oxLDL, or OM. NFAT activity was modulated using the NFAT inhibitor VIVIT $(1 \mu \mathrm{mol} / \mathrm{l}, \mathbf{a}, \mathbf{b})$ and NFATc1 expression using siRNA and a control siRNA (siCon) (c-f). a,d Matrix mineralisation. b,f RUNX2 mRNA expression. c NFATcl mRNA expression. e ALP activity; $n=3-6$; ${ }^{*} p<0.05$ vs con, ${ }^{\dagger} p<0.05$; by oneway ANOVA. Results are given as mean \pm SEM 
siRNA and achieved a stable NFATc1 reduction of $60-80 \%$ over 21 days (data not shown, Fig. 3c). Knockdown of NFATC1 prevented oxLDL-induced osteogenic transformation as assessed by matrix mineralisation (Fig. 3d), ALP activity (Fig. 3e) and RUNX2 expression (Fig. 3f). Notably, NFATc1 inhibition by siRNA did not alter vascular calcification induced by OM (Fig. 3d,e).

Because vascular calcification shares some molecular processes with osteoblast differentiation, we assessed whether the NFAT pathway plays a role in oxLDLpromoted osteoblast mineralisation. oxLDL significantly induced NFATC1 and NFATC2 mRNA expression in mature osteoblasts (Fig. 4a). OxLDL-promoted osteoblast mineralisation was prevented by inhibition of NFAT activity using VIVIT (Fig. 4b) as well as NFATc1 knockdown (Fig. 4c), in which NFATc1 was reduced by $70 \%$ (Fig. 4d). Interestingly, osteoblast mineralisation per se was not altered by modulation of the NFAT pathway.

Involvement of ROS in oxLDL-induced osteogenic transdifferentiation of HCASMCs To determine the role of ROS in the process of HCASMC mineralisation, we used the antioxidant NAC. NAC completely prevented oxLDL-induced matrix mineralisation (Fig. 5a) and RUNX2 expression (Fig. 5b), whereas it had no effect in control conditions.

OxLDL-conditioned medium of HCASMCs promotes osteoblast mineralisation Next, we assessed the paracrine vascular smooth muscle cell-bone cell interaction by analysing the
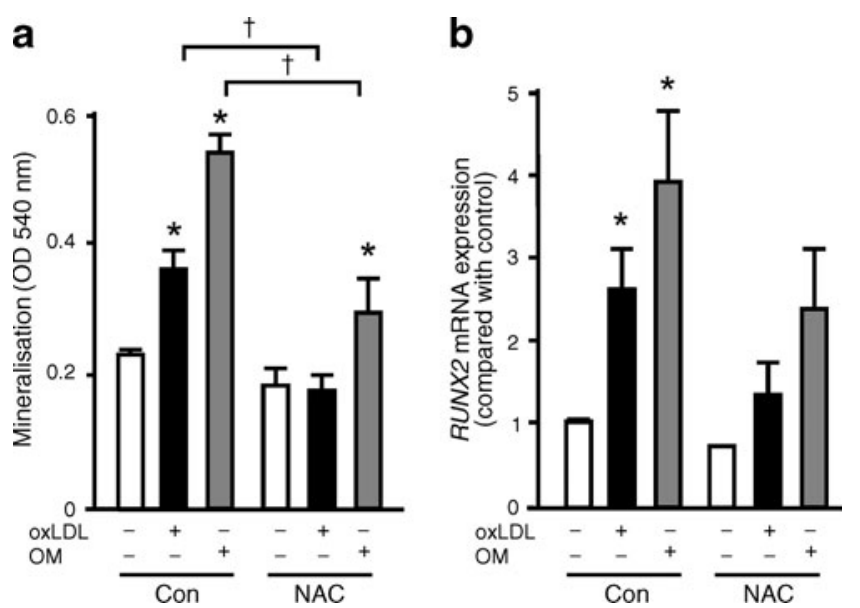

Fig. 5 ROS are involved in oxLDL-induced osteoblast mineralisation. HCASMCs were cultured for 21 days in control medium (Con), control medium supplemented with $50 \mu \mathrm{g} / \mathrm{ml}$ oxLDL, or OM. The radical scavenger NAC $(500 \mu \mathrm{mol} / 1, \mathbf{a}, \mathbf{b})$ was added during the whole transdifferentiation period. a Matrix mineralisation. b $R U N X 2$ expression. $n=3-6 ;{ }^{*} p<0.05$ vs Con, ${ }^{\dagger} p<0.05$; by one-way ANOVA

potential of conditioned HCASMC medium to modulate osteoblast mineralisation. The supernatant fraction of HCASMCs that had been treated with $50 \mu \mathrm{g} / \mathrm{ml}$ oxLDL for 21 days significantly increased osteoblast matrix mineralisation by 3.2 -fold compared with the supernatant fraction obtained from the untreated control (Fig. 6a). This increased osteoblast matrix mineralisation was prevented by using the supernatant fraction of HCASMCs that had been treated with oxLDL and VIVIT for 21 days a

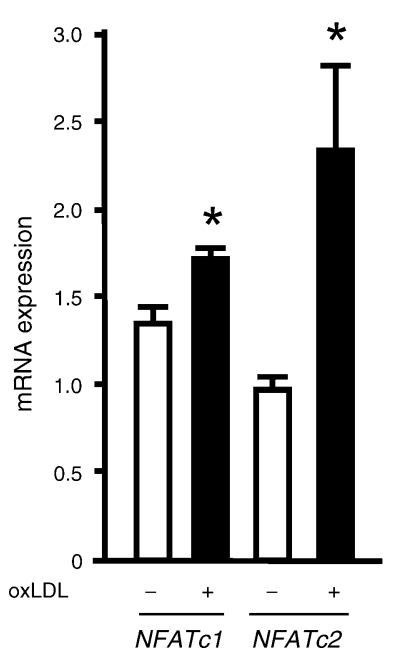

b
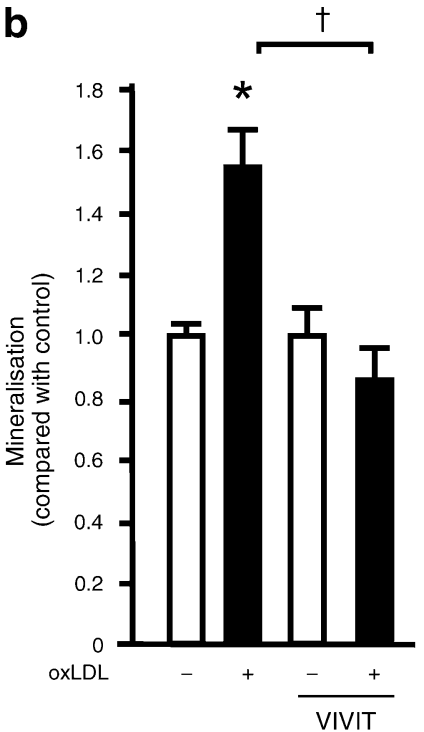

Fig. 4 The NFAT pathway is involved in oxLDL-induced osteoblast mineralisation. BMSCs were differentiated into mature osteoblasts using OM, or OM supplemented with $50 \mu \mathrm{g} / \mathrm{ml}$ oxLDL for 21 days. a NFATcl and NFATc2 mRNA expression. NFAT activity was modulated
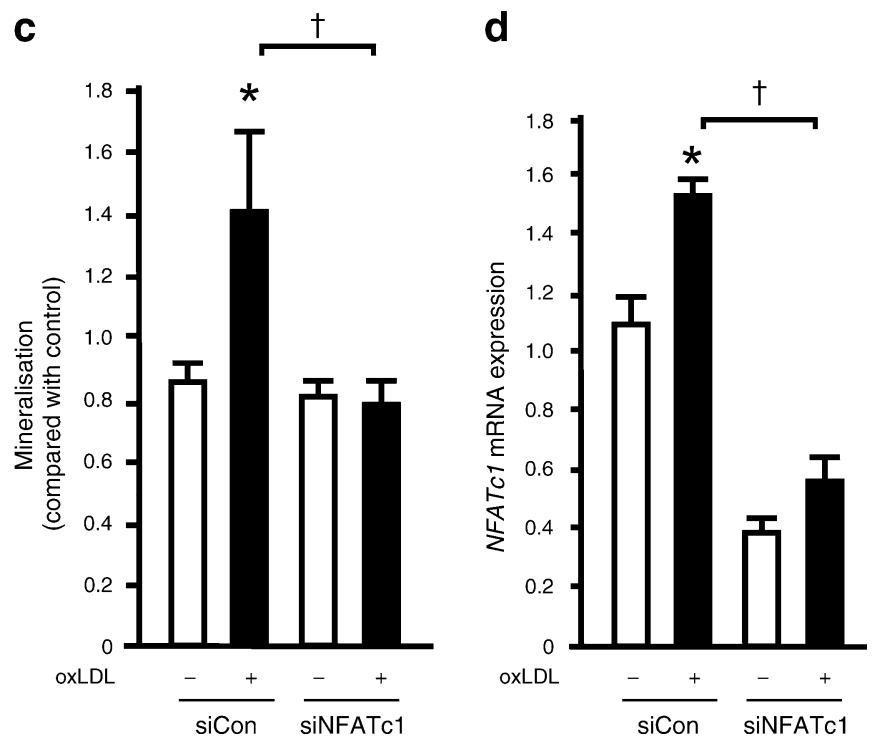

using the NFAT inhibitor VIVIT $(1 \mu \mathrm{mol} / 1, \mathbf{b})$, and NFATcl expression was modulated using siRNA (c,d). b,c Matrix mineralisation. d NFATc1 mRNA expression. $n=3-6 ;{ }^{*} p<0.05$ vs Con, ${ }^{\dagger} p<0.05$ 


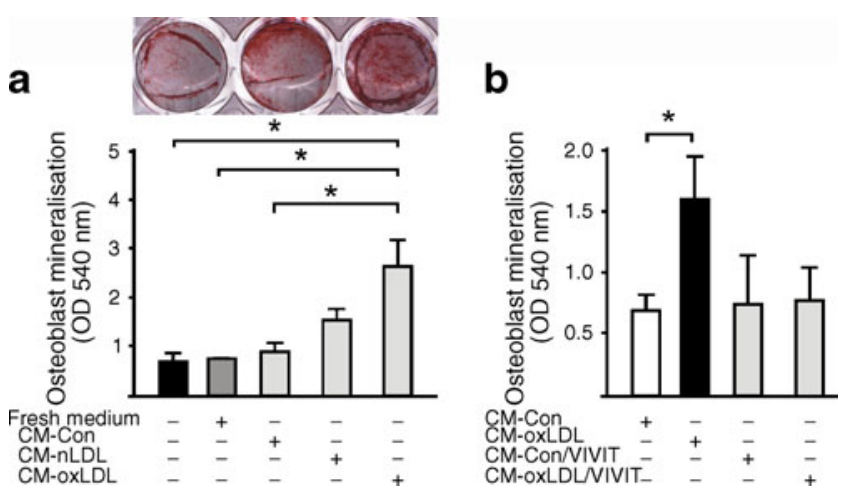

Fig. 6 HCASMC medium conditioned with oxLDL promotes osteoblast differentiation. BMSCs were grown for 21 days in osteoblast differentiation medium $(50 \%)$ as well as $50 \%$ conditioned medium $(\mathrm{CM})$ obtained from HCASMCs that had been treated with $50 \mu \mathrm{g} / \mathrm{ml} \mathrm{nLDL}$ or oxLDL (CM-nLDL, CM-oxLDL) without (a) or with (b) the NFAT inhibitor VIVIT. Conditioned medium obtained from untreated cells (CM-Con) and fresh medium, which had never been used for cell culture, served as controls. Matrix mineralisation was assessed by Alizarin Red S staining. Representative Alizarin S staining is shown in the upper part. Supernatant fractions from five independent experiments and two different batches of precursor cells were used. ${ }^{*} p<0.05$ by ANOVA. Results are given as mean \pm SEM

(Fig. 6b). Fresh HCASMC medium, as well as conditioned HCASMC control medium, did not alter osteoblast matrix mineralisation.

Elevated oxLDL levels are associated with increased vascular calcium in vivo To confirm our data in an in vivo model, we used the ZDF rat, an established rodent model of type 2 diabetes mellitus. As expected, ZDF rats had elevated blood glucose level and increased variables of lipid metabolism (Table 1). Of note, we observed an increased electrophoretic mobility of LDL, reflecting the enhanced oxidation of LDL in diabetic ZDF rats (Fig. 7a). In addition, conjugated diene formation was twofold higher in LDL from ZDF rats compared with WT rats (Fig. 7b).

Table 1 Biochemical analyses of serum variables in WT and ZDF rats

\begin{tabular}{lrrr}
\hline Variable & \multicolumn{1}{l}{ WT } & \multicolumn{1}{l}{ ZDF } & $p$ value \\
\hline Glucose (mmol/l) & $20.18 \pm 0.81$ & $38.84 \pm 1.07$ & $<0.001$ \\
Triacylglycerol (mmol/l) & $1.05 \pm 0.15$ & $5.65 \pm 0.64$ & $<0.001$ \\
Cholesterol (mmol/l) & $2.30 \pm 0.08$ & $6.51 \pm 0.36$ & $<0.001$ \\
LDL (mmol/l) & $0.35 \pm 0.02$ & $0.86 \pm 0.09$ & $<0.001$ \\
Calcium (mmol/l) & $2.51 \pm 0.04$ & $2.68 \pm 0.04$ & 0.013 \\
Phosphate (mmol/l) & $2.29 \pm 0.03$ & $2.43 \pm 0.19$ & 0.514 \\
Ca $\times$ P product & $5.75 \pm 0.11$ & $6.45 \pm 0.44$ & 0.177 \\
\hline
\end{tabular}

$n=7-8$. Data are mean \pm SEM
Fig. 7 oxLDL levels are associated with increased vascular calcium in diabetic rats. Thoracic aortas were isolated from WT and ZDF rats and serum samples were analysed. a The oxidation of LDL was assessed by agarose gel electrophoresis. As a control, in vitro oxidation of human nLDL (lane 1) to oxLDL (lane 2) is shown. b Conjugated diene formation $(n=3, * p<0.05)$. c Calcium and phosphate content of the aorta $\left(n=3,{ }^{*} p<0.05\right)$. mRNA expression of aortic (d) Runx2, Opn and Bmp2, (e) Nfatc1, and (f) Nox1 and Nox4 $\left(n=6,{ }^{*} p<0.05\right)$. $\mathbf{c}, \mathbf{d}, \mathbf{f}$ White bars, WT; black bars, ZDF. $\mathbf{g}$ Correlation of calcium deposition in the aortic wall with bone mineral density (BMD; white squares, dotted line; $r=-0.87 ; p=0.024 ; n=6$ ) and the bone resorption marker type I collagen C-telopeptide (CTX; black squares, solid line; $r=0.67 ; p=0.048 ; n=6)$. Results are given as mean $\pm \mathrm{SEM}$

The thoracic aortas isolated from ZDF rats showed significantly higher calcium and phosphate levels (Fig. 7c) compared with WT rats, as well as an increased $\mathrm{Ca} \times \mathrm{P}$ product (WT 4.24 $\pm 0.58 ;$ ZDF 9.63 $\pm 1.24 ; p=0.017$ ). Consistent with these findings, the aortic vessel from diabetic rats showed features of osteogenic transformation. Aortic mRNA expression of Runx2, Opn (also known as Spp1) and Bmp2 were significantly increased in diabetic rats (Fig. 7d). Osterix (SP7), another osteoblastic transcription factor, tended to be expressed more highly in ZDF rats (WT $0.65 \pm 0.22$; ZDF $2.82 \pm 1.32, n=5, p=0.145$ ). Aortic expression of $\mathrm{Natcl}$ was 3.4-fold higher in diabetic compared with non-diabetic rats (Fig. 7e). In addition, VSMCs isolated from ZDF rats showed a trend towards higher osteogenic transformation based on matrix mineralisation $\left(\mathrm{OD}_{540}\right.$ : WT $0.18 \pm 0.04$; ZDF $0.25 \pm 0.03 ; n=4-5 ; p=0.055$ ).

Since ROS plays a role in aortic calcification, we analysed the expression of NADPH oxidase subunits, as the main ROS building enzyme in the aorta. While Noxl was significantly downregulated in diabetic compared with non-diabetic rats, the aortic mRNA expression of Nox4 was 4.4-fold higher (Fig. 7f).

Because an elevated $\mathrm{Ca} \times \mathrm{P}$ product in the circulation may contribute to vascular calcification, total serum calcium and phosphate were measured in the rats. ZDF rats exhibited slightly higher serum calcium concentrations than WT rats (Table 1), but the calculated $\mathrm{Ca} \times \mathrm{P}$ product as well as serum creatinine levels were similar between WT and ZDF rats, thus excluding advanced diabetic renal insufficiency as an underlying mechanism.

Since bone loss and vascular calcification are closely linked, we analysed the correlation of determinants of bone metabolism and the vascular calcium content in WT and ZDF rats. The total bone mineral density of the fourth lumbar (L4) vertebrae was negatively correlated with aortic calcium content $(r=-0.87 ; p=0.024 ; n=6 ;$ Fig. $7 \mathrm{~g})$. Furthermore, serum type I collagen C-telopeptide (CTX), a bone resorption marker, was positively correlated with aortic calcium deposition $(r=0.67 ; p=0.048 ; n=6$; Fig. $7 \mathrm{~g})$. 
a
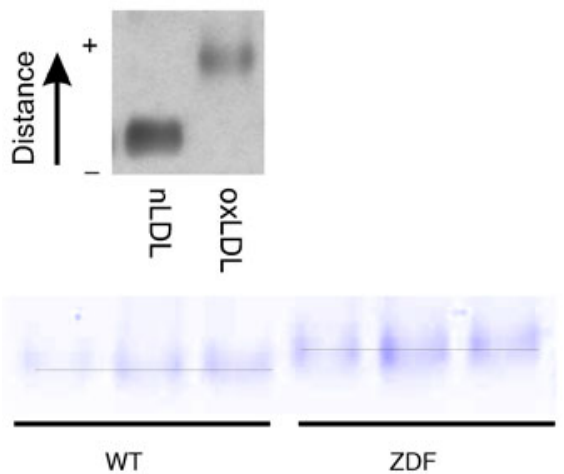

d

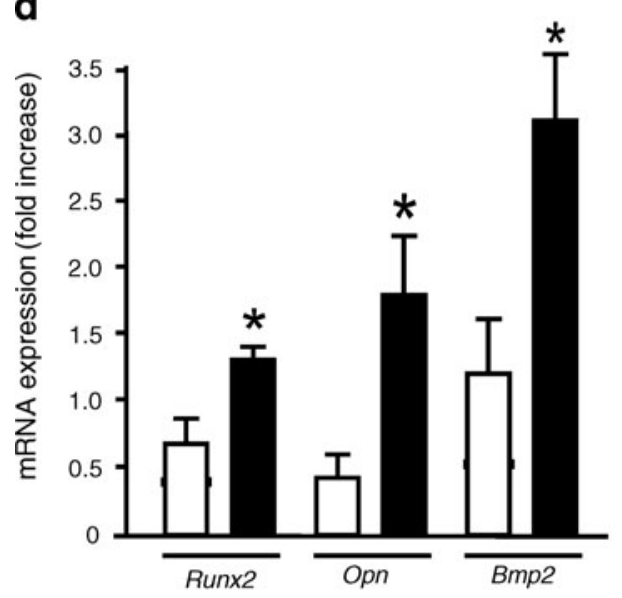

b

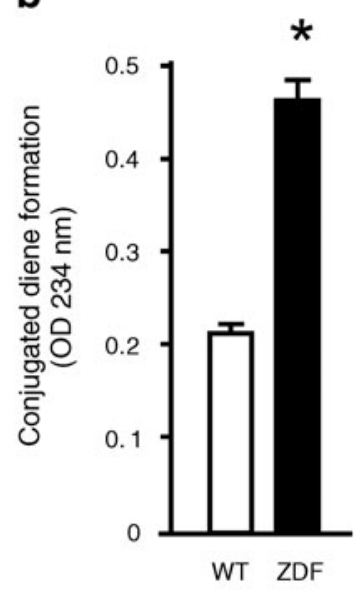

e

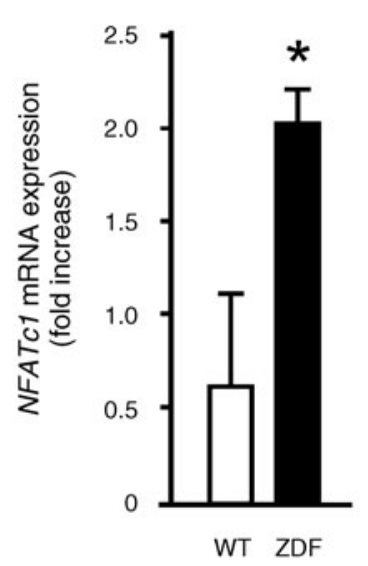

C

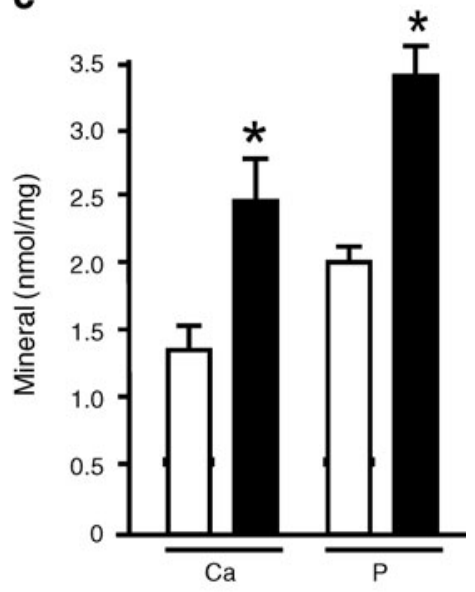

f

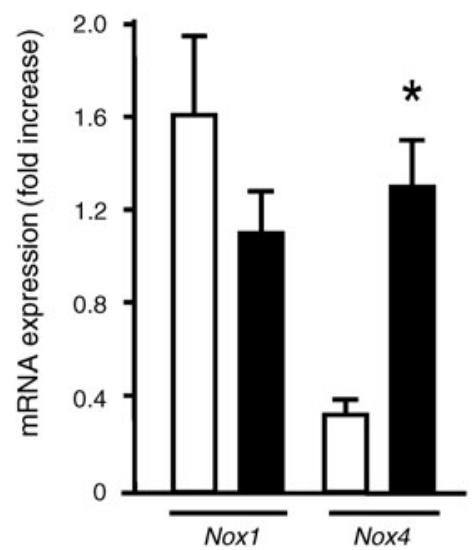

g

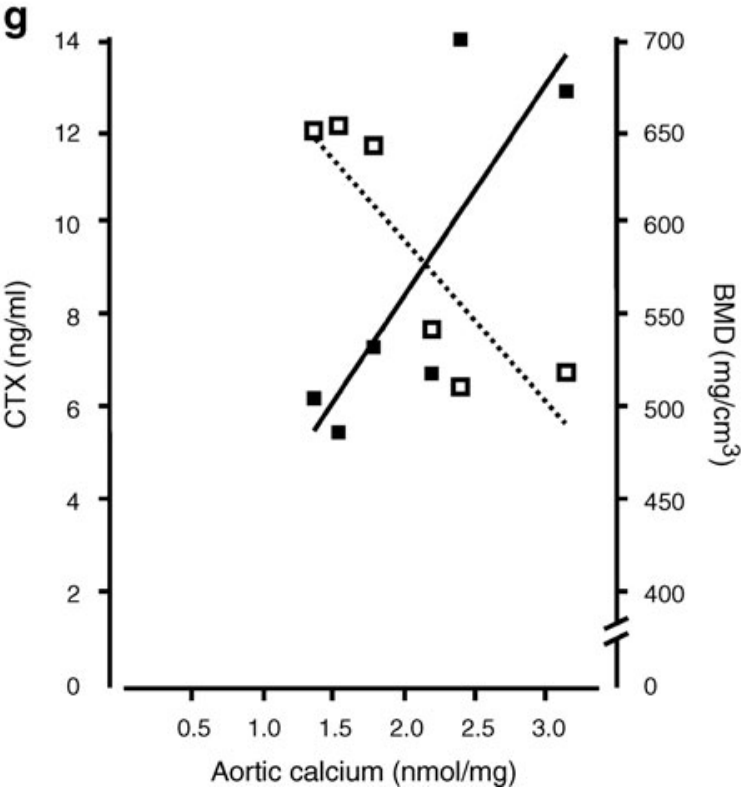




\section{Discussion}

We report for the first time that the NFAT signalling pathway is involved in vascular calcification induced by oxLDL. Inhibition of NFAT activity completely prevented oxLDLinduced osteogenic transformation of HCASMCs. The underlying mechanism involves the activation of NFAT by oxLDL, which has also been reported for Jurkat cells [24]. Upon activation, NFAT is able to bind to the RUNX2 promoter and increases transcriptional activation of RUNX2. In fact, oxLDL-increased RUNX2 promoter activity can be blocked by VIVIT (data not shown). Surprisingly, using OM, the HCASMC matrix mineralisation was not altered, whereas RUNX2 expression was inhibited by NFAT inhibition. In osteoblasts, dexamethasone stabilises RUNX2 at the protein level to allow a more efficient induction of an osteoblastic phenotype (M. Rauner et al., unpublished data). This mechanism may also apply to HCASMCs and thereby neutralise the effect of decreased transcriptional regulation of RUNX2 by NFAT inhibition. The transcription factor NFAT was shown to be involved in diabetic kidney disease [30] and was described as a metabolic sensor for the arterial VSMC response to high glucose [31, 32]. In addition, the therapeutic potential of NFAT inhibition has been demonstrated in cardiovascular disorders, including injury-induced vascular wall remodelling [33], neointima formation [34] and cardiac hypertrophy [25]. In light of our data and the predominant expression of NFATc1 in stenotic valves adjacent to calcified areas [26], NFAT inhibition might be a therapeutic strategy to prevent cellular transdifferentiation during vascular calcification induced by oxidative stress.

oxLDL-induced calcification of HCASMCs is redox sensitive, since scavenging of ROS using the antioxidant NAC blocks matrix mineralisation of HCASMCs. Furthermore, using $\mathrm{OM}, \mathrm{NAC}$ also inhibited mineralisation and $R U N X 2$ expression, which means that some compound in the OM generates ROS and, by this mechanism, initiates mineralisation. In fact, glucocorticoids are able to augment ROS production in the vascular system [35, 36]. ROS, in particular hydrogen peroxide, may potentiate aortic valve calcification [7]. In addition $\beta$-glycerol-phosphate-induced oxidative stress was associated with $R U N X 2$ expression followed by $A L P$ (also known as PDLIM3) expression and activity in A7r5 rat VSMCs [37]. VSMCs contain numerous sources of ROS, including the NADPH oxidases (NOX), xanthine oxidase, lipoxygenases and nitric oxide synthases [38]. Enhanced ROS formation in response to oxLDL can be completely abrogated by the Nox inhibitor VAS2870 [39]. Thus we evaluated whether Nox1, the main NADPH oxidase isoform in VSMCs, is increased in calcified HCASMCs. Our results were unexpected, as NOX1 was decreased in oxLDL-induced osteogenic differentiated HCASMCs (C. Goettsch, unpublished observa- tion). We conclude that NOX1-mediated ROS formation does not play a role in in vitro vascular calcification by oxLDL. This is in line with a recent study showing decreased levels of Nox isoforms in calcified aortic valves due to reduced activity of antioxidative enzymes and increased activity of uncoupled nitric oxide synthase [40]. In the aorta of diabetic rats, we observed a switch from NOX1 to NOX4 activity. Recently, S100A12-induced calcification of VSMCs was found to be mediated by oxidative stress involving NOX [41]. While NOX1 and NOX2 generate superoxide, NOX4 produces hydrogen peroxide [42] and can thereby act as a switch from proliferation to differentiation of pre-adipocytes [43]. Our data and the published data suggest that this NOX balance is critical for transdifferentiation of VSMCs during vascular calcification.

Emerging evidence supports the concept of bi-directional crosstalk between bone cells and cells of the vascular system in the common pathogenesis of osteoporosis and vascular calcification. Treatment of human RANKL knockin mice with denosumab, a monoclonal antibody against RANKL, inhibits glucocorticoid-induced loss of bone mass [4] and vascular calcium deposition [5]. In our in vivo study using diabetic rats, we found a negative correlation between bone mass and vascular calcium content and a positive correlation between bone resorption markers and vascular calcium content, suggesting that vascular calcium most likely originates from bone. Moreover, there is emerging evidence that vascular calcification and osteoblast matrix mineralisation share some molecular and cellular processes. Indeed, oxLDL promoted osteoblast matrix mineralisation, which was prevented by NFAT inhibition. Our co-culture experiments underline the functional relevance of, and demonstrate a further link between, the vascular and skeletal systems. oxLDL-conditioned HCASMC medium significantly increased osteoblast matrix mineralisation. In contrast to our findings, minimally oxidised LDL inhibits the differentiation of pre-osteoblast cell lines [19, 20]. Reasons for these opposite effects could be the use of human primary cells and the concentration of oxLDL used, which was 2.5to 4-fold lower in our experiments. In addition to osteoblasts, osteoclast-like cells were found within the calcified vessel wall [7], and may be involved in resorption of calcium deposits [44]. In fact, we and others found an oxLDLmediated inhibition of RANKL-induced osteoclast differentiation (C. Goettsch, unpublished data) [45]. Thus, the alterations to the local microenvironment may favour bone formation in calcified vascular lesions.

ZDF rats are models for human type 2 diabetes mellitus with hyperglycaemia, dyslipidaemia, insulin resistance and endothelial dysfunction [46]. Diabetic rats with higher oxLDL serum levels displayed higher aortic calcium content compared with normal rats. In fact, the Multi- 
Ethnic Study of Atherosclerosis demonstrated an association between high oxLDL and the severity of coronary calcification [47]. Furthermore, enhanced circulating levels of oxLDL have been found in patients with impaired glucose tolerance [17] and type 2 diabetes [18]. In our study, VSMCs isolated from diabetic rats tended to become easily calcified. In line with our findings, which showed a higher aortic expression of osteoblast markers in diabetic rats, diet-induced diabetes promotes the expression of osteoblastic genes in the aorta of LDL-receptor (LDLR) knockout mice [48].

In summary, our results identify the NFAT signalling pathway as a novel regulator of oxLDL-induced transdifferentiation of VSMCs towards an osteoblast-like phenotype. Therefore, the transcription factor NFAT may evolve as a target to prevent vascular calcification in diabetes mellitus and conditions with enhanced oxidative stress.

Acknowledgements We thank N. Pacyna and B. Zeiler for their excellent technical assistance. This work was supported by the MeDDrive33 programme of the Medical Faculty of the Technical University of Dresden, Germany, the Foundation Dresdner Herz-Kreislauftage and a grant from DFG (GO1801/4-1) to C. Goettsch, the Elsbeth Bonhoff Foundation to C. Goettsch, C. Hamann and L.C. Hofbauer and a grant from DFG/SFB TR 67 to L.C. Hofbauer and U. Hempel.

All authors had substantial contribution to: conception and design, or analysis and interpretation of data; drafting the article or revising it critically for important intellectual content; and final approval of the version to be published.

Duality of interest statement The authors declare that there is no duality of interest associated with this manuscript.

\section{References}

1. Hofbauer LC, Brueck CC, Shanahan CM, Schoppet M, Dobnig H (2007) Vascular calcification and osteoporosis - from clinical observation towards molecular understanding. Osteoporos Int 18:251-259

2. Demer LL, Tintut Y (2009) Mechanisms linking osteoporosis with cardiovascular calcification. Curr Osteoporos Rep 7:42-46

3. Jeffcoate WJ, Rasmussen LM, Hofbauer LC, Game FL (2009) Medial arterial calcification in diabetes and its relationship to neuropathy. Diabetologia 52:2478-2488

4. Hofbauer LC, Zeitz U, Schoppet M et al (2009) Prevention of glucocorticoid-induced bone loss in mice by inhibition of RANKL. Arthritis Rheum 60:1427-1437

5. Helas S, Goettsch C, Schoppet M et al (2009) Inhibition of receptor activator of NF-kappaB ligand by denosumab attenuates vascular calcium deposition in mice. Am J Pathol 175:473-478

6. Duer MJ, Friscic T, Proudfoot D et al (2008) Mineral surface in calcified plaque is like that of bone: further evidence for regulated mineralization. Arterioscler Thromb Vasc Biol 28:2030-2034

7. Liberman M, Bassi E, Martinatti MK et al (2008) Oxidant generation predominates around calcifying foci and enhances progression of aortic valve calcification. Arterioscler Thromb Vasc Biol 28:463-470

8. Panizo S, Cardus A, Encinas M et al (2009) RANKL increases vascular smooth muscle cell calcification through a RANKBMP4-dependent pathway. Circ Res 104:1041-1048
9. Aikawa E, Nahrendorf M, Figueiredo JL et al (2007) Osteogenesis associates with inflammation in early-stage atherosclerosis evaluated by molecular imaging in vivo. Circulation 116:28412850

10. Hofbauer LC, Schoppet M (2004) Clinical implications of the osteoprotegerin/RANKL/RANK system for bone and vascular diseases. JAMA 292:490-495

11. Steitz SA, Speer MY, Curinga G et al (2001) Smooth muscle cell phenotypic transition associated with calcification: upregulation of Cbfa1 and downregulation of smooth muscle lineage markers. Circ Res 89:1147-1154

12. Tintut Y, Patel J, Parhami F, Demer LL (2000) Tumor necrosis factor-alpha promotes in vitro calcification of vascular cells via the cAMP pathway. Circulation 102:2636-2642

13. Hofbauer LC, Schrader J, Niebergall U et al (2006) Interleukin-4 differentially regulates osteoprotegerin expression and induces calcification in vascular smooth muscle cells. Thromb Haemost 95:708-714

14. Speer MY, Yang HY, Brabb T et al (2009) Smooth muscle cells give rise to osteochondrogenic precursors and chondrocytes in calcifying arteries. Circ Res 104:733-741

15. Ambasta RK, Kumar P, Griendling KK, Schmidt HH, Busse R, Brandes RP (2004) Direct interaction of the novel Nox proteins with $\mathrm{p} 22$ phox is required for the formation of a functionally active NADPH oxidase. J Biol Chem 279:45935-45941

16. Goettsch C, Goettsch W, Muller G, Seebach J, Schnittler HJ, Morawietz H (2009) Nox4 overexpression activates reactive oxygen species and p38 MAPK in human endothelial cells. Biochem Biophys Res Commun 380:355-360

17. Kopprasch S, Pietzsch J, Kuhlisch E et al (2002) In vivo evidence for increased oxidation of circulating LDL in impaired glucose tolerance. Diabetes 51:3102-3106

18. Kopprasch S, Pietzsch J, Ansurudeen I et al (2009) Prediabetic and diabetic in vivo modification of circulating low-density lipoprotein attenuates its stimulatory effect on adrenal aldosterone and cortisol secretion. J Endocrinol 200:45-52

19. Parhami F, Morrow AD, Balucan J et al (1997) Lipid oxidation products have opposite effects on calcifying vascular cell and bone cell differentiation. A possible explanation for the paradox of arterial calcification in osteoporotic patients. Arterioscler Thromb Vasc Biol 17:680-687

20. Mody N, Parhami F, Sarafian TA, Demer LL (2001) Oxidative stress modulates osteoblastic differentiation of vascular and bone cells. Free Radic Biol Med 31:509-519

21. Bear M, Butcher M, Shaughnessy SG (2008) Oxidized lowdensity lipoprotein acts synergistically with beta-glycerophosphate to induce osteoblast differentiation in primary cultures of vascular smooth muscle cells. J Cell Biochem 105:185-193

22. Yan J, Stringer SE, Hamilton A et al (2011) Decorin GAG synthesis and TGF-beta signaling mediate Ox-LDL-induced mineralization of human vascular smooth muscle cells. Arterioscler Thromb Vasc Biol 31:608-615

23. Flanagan WM, Corthesy B, Bram RJ, Crabtree GR (1991) Nuclear association of a $\mathrm{T}$ cell transcription factor blocked by FK-506 and cyclosporin A. Nature 352:803-807

24. Maziere C, Morliere P, Massy Z et al (2005) Oxidized low-density lipoprotein elicits an intracellular calcium rise and increases the binding activity of the transcription factor NFAT. Free Radic Biol Med 38:472-480

25. Yu H, van Berkel TJ, Biessen EA (2007) Therapeutic potential of VIVIT, a selective peptide inhibitor of nuclear factor of activated T cells, in cardiovascular disorders. Cardiovasc Drug Rev 25:175-187

26. Alexopoulos A, Bravou V, Peroukides S et al (2010) Bone regulatory factors NFATc1 and Osterix in human calcific aortic valves. Int J Cardiol 139:142-149 
27. Rauner M, Sipos W, Goettsch C et al (2009) Inhibition of lamin A/C attenuates osteoblast differentiation and enhances RANKLdependent osteoclastogenesis. J Bone Miner Res 24:78-86

28. Rauner M, Goettsch C, Stein N et al (2011) Dissociation of osteogenic and immunological effects by the selective glucocorticoid receptor agonist, compound $\mathrm{A}$, in human bone marrow stromal cells. Endocrinology 152:103-112

29. Aramburu J, Yaffe MB, Lopez-Rodriguez C, Cantley LC, Hogan PG, Rao A (1999) Affinity-driven peptide selection of an NFAT inhibitor more selective than cyclosporin A. Science 285:2129-2133

30. Sanchez AP, Sharma K (2009) Transcription factors in the pathogenesis of diabetic nephropathy. Expert Rev Mol Med 11:e13

31. Nilsson-Berglund LM, Zetterqvist AV, Nilsson-Ohman J et al (2010) Nuclear factor of activated T cells regulates osteopontin expression in arterial smooth muscle in response to diabetes-induced hyperglycemia. Arterioscler Thromb Vasc Biol 30:218-224

32. Nilsson J, Nilsson LM, Chen YW, Molkentin JD, Erlinge D, Gomez MF (2006) High glucose activates nuclear factor of activated $\mathrm{T}$ cells in native vascular smooth muscle. Arterioscler Thromb Vasc Biol 26:794-800

33. Karpurapu M, Wang D, van Quyen D et al (2010) Cyclin D1 is a bona fide target gene of NFATc1 and is sufficient in the mediation of injury-induced vascular wall remodeling. J Biol Chem 285:3510-3523

34. Liu Z, Zhang C, Dronadula N, Li Q, Rao GN (2005) Blockade of nuclear factor of activated $\mathrm{T}$ cells activation signaling suppresses balloon injury-induced neointima formation in a rat carotid artery model. J Biol Chem 280:14700-14708

35. Schafer SC, Wallerath T, Closs EI et al (2005) Dexamethasone suppresses eNOS and CAT-1 and induces oxidative stress in mouse resistance arterioles. Am J Physiol Heart Circ Physiol 288:H436-H444

36. Muzaffar S, Shukla N, Angelini GD, Jeremy JY (2005) Prednisolone augments superoxide formation in porcine pulmonary artery endothelial cells through differential effects on the expression of nitric oxide synthase and NADPH oxidase. Br J Pharmacol 145:688-697

37. Sutra T, Morena M, Bargnoux AS, Caporiccio B, Canaud B, Cristol JP (2008) Superoxide production: a procalcifying cell signalling event in osteoblastic differentiation of vascular smooth muscle cells exposed to calcification media. Free Radic Res 42:789-797

38. Clempus RE, Griendling KK (2006) Reactive oxygen species signaling in vascular smooth muscle cells. Cardiovasc Res $71: 216-225$

39. Stielow C, Catar RA, Muller G et al (2006) Novel Nox inhibitor of oxLDL-induced reactive oxygen species formation in human endothelial cells. Biochem Biophys Res Commun 344:200-205

40. Miller JD, Chu Y, Brooks RM, Richenbacher WE, Pena-Silva R, Heistad DD (2008) Dysregulation of antioxidant mechanisms contributes to increased oxidative stress in calcific aortic valvular stenosis in humans. J Am Coll Cardiol 52:843-850

41. Hofmann Bowman MA, Gawdzik J, Bukhari U et al (2011) S100A12 in vascular smooth muscle accelerates vascular calcification in apolipoprotein E-null mice by activating an osteogenic gene regulatory program. Arterioscler Thromb Vasc Biol 31:337-344

42. Takac I, Schroder K, Zhang L et al (2011) The E-loop is involved in hydrogen peroxide formation by the NADPH oxidase Nox4. J Biol Chem 286:13304-13313

43. Schroder K, Wandzioch K, Helmcke I, Brandes RP (2009) Nox4 acts as a switch between differentiation and proliferation in preadipocytes. Arterioscler Thromb Vasc Biol 29:239-245

44. Bas A, Lopez I, Perez J, Rodriguez M, Aguilera-Tejero E (2006) Reversibility of calcitriol-induced medial artery calcification in rats with intact renal function. J Bone Miner Res 21:484-490

45. Maziere C, Louvet L, Gomila C, Kamel S, Massy Z, Maziere JC (2009) Oxidized low density lipoprotein decreases Rankl-induced differentiation of osteoclasts by inhibition of Rankl signaling. J Cell Physiol 221:572-578

46. Clark JB, Palmer CJ, Shaw WN (1983) The diabetic Zucker fatty rat. Proc Soc Exp Biol Med 173:68-75

47. Holvoet P, Jenny NS, Schreiner PJ, Tracy RP, Jacobs DR (2007) The relationship between oxidized LDL and other cardiovascular risk factors and subclinical CVD in different ethnic groups: the Multi-Ethnic Study of Atherosclerosis (MESA). Atherosclerosis 194:245-252

48. Towler DA, Bidder M, Latifi T, Coleman T, Semenkovich CF (1998) Diet-induced diabetes activates an osteogenic gene regulatory program in the aortas of low density lipoprotein receptor-deficient mice. J Biol Chem 273:30427-30434 